\title{
Improving Collaborative Learning Using Pervasive Embedded System-Based Multi-Agent Information and Retrieval Framework in Educational Systems
}

\author{
A.A. Ileladewa, UniversitiTunku Abdul Rahman (UTAR)
}

\begin{abstract}
E-learning is a form of Technology Supported Education where the medium of instruction is through Digital Technologies, particularly Computer Technology. An instance is the use of search engines like Google and Yahoo, which aid Collaborative Learning. However, the widespread provision of distributed, semi-structured information resources such as the Web has obviously brought a lot of benefits; but it also has a number of difficulties. These difficulties include people getting overwhelmed by the sheer amount of information available, making it hard for them to filter out the junk and irrelevancies and focus on what is important, and also to actively search for the right information. Also, people easily get bored or confused while browsing the Web because of the hypertext nature of the web, while making it easy to link related documents together, it can also be disorienting. To alleviate these problems, the Web Information Food Chain Model was introduced. How effective has this been with the dynamic nature of computing technologies? Pervasive computing devices enable people to gain immediate access to information and services anywhere, anytime, without having to carry around heavy and impractical computing devices. Thus, the bulky PCs become less attractive and being slowly eroded with the development of a new generation of smart devices like wireless PDAs, smart phones, etc. These embedded devices are characterized by being unobtrusively embedded; completely connected; intuitively intelligent; effortlessly portable and mobile; and constantly on and available. This paper presents the use of embedded systems and Intelligent Agent-Based Web Information Food Chain Model in Multi-Agent Information and Retrieval Framework (IIFCEMAF), to realizing full potentials of the internet, for users' improved system of collaborative e-learning in education.
\end{abstract}

Keywords: Collaborative Learning; Pervasive Computing; Multi-Agent System; Information Food Chain

\section{INTRODUCTION}

The open Internet and worldwide Web allow us to access multimedia data and knowledge located throughout the world.
Clearly, these new technologies present enormous opportunities for posting, finding, organizing and sharing vast amounts of information. This is the premise of intelligent information agents which can play a dominant role in our evolving information infrastructure, if they are proven to be useful to people, organizations and enterprises for intelligent information search and management. Multi-Agent System (MAS) refers to an arrangement where multiple agents communicate with each other, observe and act upon the environment, which directs its activity towards achieving certain goals or interests [3].MAS is being widely used in many systems nowadays, whether it is an online application or offline system. The main task of such agents is to perform pro-active searches for, maintain, and mediate relevant information on behalf of their users or other agents. This includes skills such as retrieving, analyzing, manipulating, fusing heterogeneous information as well as visualizing of and guiding the user through the available, individual information space, which definitely will also aid an improved Collaborative Learning.

Collaborative Learning is a situation in which two or more people learn or attempt to learn something together[6].Unlike individual learning, people engaged in collaborative learning capitalize on one another's resources and skills (asking one another for information, evaluating one another's ideas, monitoring one another's work etc.) [4]. Furthermore, collaborative learning is based on the model that knowledge can be created within a population where members actively interact by sharing experiences and take on asymmetry roles [15].Thus, collaborative learning is commonly illustrated when groups of students work together to search for understanding, meaning, or solutions or to create an artifact or product of their learning.Examples include Collaborative Networked Learning (CNL), which is a form of collaborative learning for the selfdirected adult learners; Computer-Supported Collaborative Learning (CSCL), a relatively new educational paradigm within collaborative learning which uses technology in a learning environment to help mediate and support group interactions in a collaborative learning context [15]; Collaborative Learning Development (CLD), which enables developers of learning systems to work as a network. Precisely, this is relevant to elearning where developers can share and build knowledge into courses in a collaborative environment. Knowledge of a single subject can be pulled together from remote locations using software systems. These, and various other educative 
application systems would definitely have to be mounted on the web for collaborative learning to take place amongst their respective target learners, who are assumed to be connected to the internet.

From the foregoing, it shows clearly that only a good e-learning system, can guarantee a good collaborative learning since the information source as it applies to each of the subject applications must be mounted on the Web regardless of the number of target users. In spite of the useful benefits presented by the Web, the reality of its use since the beginning of the $21^{\text {st }}$ century is that it is somewhat being beset by some problems even though they are becoming relatively reduced in the recent time. The problems include: 'information overload'- which makes people to get overwhelmed by the sheer amount of information available, making it hard for them to filter out the junk and irrelevancies and focus on what is important, and also to actively search for the right information [17]; people getting easily bored or confused while browsing the Web because of the hypertext nature of the web, while making it easy to link related documents together, it can also be disorienting, hence getting lost in cyberspace becomes so easy (that is, it is equally easy for people to either miss or misunderstand things when searching for a particular item of information; and lastly, the Web was not really designed to be used in a methodical way, coupled with most Web pages tending to be attractive and highly animated at the expense of the more paramount goal of making a well-designed Web page to effectively convey precise information. This makes the Web not generally appropriate when attempting to answer a complex important query.

In order to solve the aforementioned problems, agents have widely been proposed as a solution, because Information Agent (IA ) has access to at least one and many more information sources, and is able to collate and manipulate information obtained from these sources, in order to answer queries posed by users and other IAs. It is worth noting that the information sources may be of many types, including, for example, traditional databases, as well as other information agents. However, in spite of attemptsby Search engines such as Google and Yahoo to alleviate these problems by indexing largely unstructured and unmanaged information on the web, they still tend to lack adequate functionality because their simple search features are: not tailored to a user's particular demand; directed mostly at textual contents - despite the fact the Web supports heterogeneous, multimedia content; and lastly, it is uncertain that the brute-force indexing techniques used by the current search engines will match the internet size in the next century. So, finding and managing information precisely on the internet is still a problem, despite tools such as Google and Yahoo [17]. In order to realize full potentials of the internet, including having a collaborative environment of optimal e-learning, and to overcome theseproblemsearlier stated, this framework of tools tagged "The IIFCEMAF", is hereby being proposed. The system, aims at having a single coherent view of distributed, heterogeneous information resources, providing rich, personalized, user-oriented services that ensure flexible and efficient services using these tools that are adaptive, selfoptimizing, scalable, distributed, and modular, to support the expected growth of the internet and the web.

The rest of the paper is organized as follows: Section 2 defines the problem statement, Section 3 gives the literature review of Collaborative Learning and Multi-Agent Information and Retrieval System, Section 4 describes the current typical MultiAgent System Framework (the Intelligent Agent-Based Web Information Food Chain Model and a Multi-Agent Information System Architecture), Section 5 is about Modification and Adoption of Intelligent Web Information Model and Pervasive Embedded Systems into Proposed framework of IIFCEMAF Architecture, and discussions of major concerns, for improved Collaborative Learning. Last Section is the conclusion with future work.

\section{Problem Statement}

The identified problems associated with the reality of the use of Web since the beginning of $21^{\text {st }}$ century, as highlighted in section 1 above, are of major concern to us, and crucial to improving collaborative learning. Also, in view of the development in computing technologies such as pervasive embedded systems like wireless PDAs, smart phones, etc., as well as the benefits derivable from them,this paper proposes a framework of tools -Intelligent Agent-Based Web Information Food Chain Model, and the use of pervasive embedded systems in Multi-Agent Information and Retrieval Framework (IIFCEMAF),torealizing full potentials of the internet, for users' improved collaborative e-learning of various kinds of discourse/subject applications in educational systems.

\section{Collaborative Learning and Multi-Agent Information and Retrieval System}

Having a good and reliable collaborative learning is mostly dependent on a good e-learning system. E-learning, is used interchangeably in a wide variety of contexts. In Companies, it refers to the strategies that use the company's network to deliver training course to employees.In the United States, it is defined as a planned teaching/learning experience that uses wide spectrum of technologies, mainly internet or computerbased, to reach learners. Lately, in most higher institutions of learning, it is used to define a specific mode to attend a course or programme of study where the students rarely, if ever, attend face-to-face or have on-campus access to educational facilities, because they study online [9]. The Web, hosting various educative applications/materials, is therefore a vital and valuable information resource to be more enriched. These information sources may be many, and of different types depending on the scale of learning interests for target users.

Most prominent solutions for finding relevant information on the Internet include monolithic Web indices such as Gopher 
and Harvest [2], as well as search enginesand search bots [1]. However, neither Web indices, nor search bots overcome the hard problems caused by the heterogeneity of systems, data syntax, structure, and semantics in a sufficient way. Methods to solve these types of heterogeneities concern intense data, metadata, and semantic information brokering [13] which goes beyond the capability of any search bot deployed so far on the Web. As a result, the notion of information agents was proposed and applied because of the access they have to at least one or more information sources, and are able to collate and manipulate information obtained from these sources in order to answer queries posed by users and other information. Thus, enabling users to find the precise information they really want to find, and shield them from information they do not want. This network of interoperating information sources are often referred to as intelligent and cooperative information systems. Hence, for the system to be very effective in itsinformation storage and retrieval process, a good multi-agent interactive framework is required, to bringing about collaborative learning.

\section{A. Background of Information Agent}

Information agent technology (IAT) [13] emerged as a major part of the more general intelligent software agent technology [14] recently as a response to the challenges mentioned above, from both, the technological and human user perspective. As such, IAT is an inherently interdisciplinary technology encompassing approaches, methods and tools from different research disciplines andsubfields such as artificial intelligence, advanced databases and knowledge base systems, distributed information systems, information retrieval, and human computer interaction.

The driving idea of IAT is the development, effective and efficient utilization of autonomous computational software entities, called intelligent information agents, which have access to multiple, heterogeneous and geographically distributed information sources as in the Internet or corporate Intranets. The main task of such agents is to perform pro-active searches for, maintain, and mediate relevant information on behalf of their users or other agents preferably just-in-time. This includes skills such as retrieving, analyzing, manipulating, fusing heterogeneous information as well asvisualizing of and guiding the user through the available, individual information space [16]. Today, agents are deployed in different settings, such as industrial control, Internet searching, personal assistance, network management, games, software distribution, and many others.

\section{B. Building Intelligent Information Agents}

Intelligent agents for the Internet are commonly called information agents (IA). They are special kind of so-called intelligent software agents. Thus, an IA is supposed to satisfy one or multiple of the following requirements [16].

* Information acquisition and management. It is capable of providing transparent access to one or many different information sources. Furthermore, it retrieves, extracts, analyzes, and filters data, monitors sources, and updates relevant information on behalf of its users or other agents.

* Information synthesis and presentation. The agent is able to fuse heterogeneous data and toprovide unified, multi-dimensional views on relevant information tothe user.

* Intelligent user assistance. The agent can dynamically adapt to changes in user preferences, the information, and network environment as well. Hence, it provides intelligent, interactive assistance for common users supporting their information-based business on the Internet.

Many systems of information agent have been developed or are currently under development in academic and commercial research labs, and can be classified as in [7], into the following. * Non-cooperative or cooperative information agents, depending on the ability of the agents to cooperate with each other for the execution of their tasks. Several protocols and methods are available for achieving cooperation among autonomous information agents in different scenarios, like hierarchical task delegation contracting, and decentralized negotiation. *Adaptive information agents are able to adapt themselves to changes in networks and information environments. Examples of such agents are learning personal assistants on the Web.

* Rational information agents behave utilitarian in an economic sense. They are acting and may even collaborate together to increase their own benefits. One main application domain of such kind of agents is automated trading and electronic commerce in the Internet.

*Mobile information agents are able to travel autonomously through the Internet. Such agents may enable, e.g. dynamic load balancing in large-scale networks, reduction of data transfer among information servers, applications, and migration of small business logic within medium-range corporate intranets on demand.

However, the high-level collaboration of an IA with other agents rely, for example, on service brokering, matchmaking, negotiation, and collaborative (social) filtering, whereas collaborating with its human users mainly corresponds to the application of techniques stemming from human-computer interaction and affective computing.

\section{Approaches for Building Information Agents}

Information agents can be built mostly using any of the following approaches.

* User Programming. An information agent is programmed explicitly by its user from scratch, for example by using a collection of user-programmed rules for processing information related to a particular 
task. This approach is characterized by a main problem of its requiring too much insight, understanding and effort from the user. For example, a user has to recognize opportunity for employing an agent, take initiative to create it, endow an agent with explicit knowledge and maintain the underlying rules over time.

*Knowledge-Engineering. An agent is endowed a-priori with a great deal of domain-specific background knowledge about the application and the user. The problems with this approach are that it requires

substantial efforts from knowledge engineer, the agent is highly domain-specific and its knowledge is relatively fixed, thus the agent is hardly adaptable to different application domains.

* Machine Learning. An agent automatically acquires the knowledge it needs to assist the user by applying appropriate methods from machine learning. However, the necessary condition for this approach to be applicable is that the use of the application involves highly repetitive and different behavior from different users. Adaptation of the agent to individual user preferences and habits is a clear advantage in that it, for example, offers customized results and requires even less work from user and application developer but it also raises the issue of trust between the user and its learning information agent.

\section{Implementation of Built Information Agent}

An information agent can be implemented as, invoked by or embedded in any client or server side Web-based application. Relevant techniques for implementing such applications are platform-independent signed Java applets, scripts, platformdependent ActiveX and CGI, FastCGI, and Java servlets, respectively. Access to relational databases may be realized using generic application programming interfaces such as JDBC (Java Database Connectivity) with embedded query language SQLJ, and Microsoft's ODBC (Open Database Connectivity). The same goes with the generic OKBC (Open Knowledge Base Connectivity) API for an agent to access multiple heterogeneous knowledge bases [11].

\section{Coordinating Societies of Information Agents}

The process of managing dependencies between activities of one or multiple actors, performed to achieve a goal and to avoid conflicts while having maximum concurrency, is called Coordination. It entails task decomposition, resource allocation, synchronization, group decision making, communication, and the preparation and adoption of common objectives.Various approaches for coordination strategiesexist, including multi-agent planning and decentralized negotiation protocols for different multi-agent environments. Recent works also investigate the benefits of learning to choose an appropriate coordination strategy by a single agent in a multiagent system [14]. Coordinating collaboration among information agents may follow some sort of social obligations from given or emerging joint intentions, delegation of tasks and responsibilities, or team plans [11], but the details of coordination mechanisms are discussed in [12]. Also, possible types of cooperation in multi-agent systems are discussed in [7].

\section{Adaptive Information Agents}

Adaptation of an agent to its environment can be done inan isolated manner or in collaboration with other agents by using methods for single or multi-agent learning, respectively [14]. Learning among multiple agents may be collective, whereby the agents adapt themselves in order to improve the benefits of the system. But system adaptation can even emerge without any collaboration when individual learning of one agent affects that of other agents in a beneficial way. Adaptive information agents deal with uncertain, incomplete and vague information in an efficient reliable way such that they are able to make intelligent decisions on the fly [5]. An agent may exhibit adaptive behaviour relative to a variety of internal reasoning processes concerning communication, coordination, planning, scheduling, and task execution monitoring [14]. All approaches and systems for single or multi-agent adaptation may be evaluated by different criteria; these criteria include the following:

$\square$ applied strategy such as learning by example, analogy, or discovery,

$\square$ kind of feedback and guidance for the agents by means of reinforcement, supervised or unsupervised learning,

$\square$ type of interaction among agents, human users and the multiagent system in the environment,

$\square$ purpose of learning as to improve the skills of a single agent or the whole system, and

$\square$ distribution of data and concurrent computation for adaptation in the multi-agent system.

The most popular application domain of adaptive single and multi-agent systems is currently electronic commerce and information gathering on the Web. Equally important domains are manufacturing [15], digital libraries [10], logistics, and telecommunication networks.

\section{Content-Based Filtering and User Profiling Versus Collaborative Filtering}

Content-based filtering and user-interest profiling is a common approach to tackle the information filtering problem. Items are recommended to a user according to correlations found between the items' content, for example, presence of certain keywords, features, and the given user preferences in a profile. Latter is usually generated and updated by the agent automatically by observing the user's online activities such as visiting Web pages, dealing with downloaded documents, adding or deleting bookmarks, and printing, as well as affective signals such as eye movement or gestures, and credit assignments to the agent.Initially, only a few systems of 
collaborating information agents show adaptive behaviour. This is partly due to the fact that there is still not much known about the exact relation between single and multi-agent adaptation, and vice versa. Some of the most widely used and effective techniques for collaborating adaptive agents are collaborative (social) filtering and genetic algorithms [14].

This collaborative recommendation technique is a powerful method for leveraging the information contained in user profiles. In contrast to content-based filtering, the agent rates the items chosen by its user and compares the corresponding user preference vector to that of other user's projection to the same set of items. It thenrecommends other items which have been recommended by users who share similar likes and dislikes. Hence, it has to collaborate with other agents to gain the respective knowledge. Thus, this technique essentially automates the process of "word of mouth" in a given user community. In addition, trust among users andagents is even easier to gain, since it is very difficult to manipulate the recommendations an agent makes to its user via social filtering. Some prominent examples ofcollaborating adaptive information agents systems are Amalthaea, InfoSpider,etc. [10].

\section{The Existing Multi-Agent Information System Framework}

\section{A. Intelligent Agent-Based Web Information Food Chain Model}

The information food chain is a model of information agents that add value to the underlying information infrastructure of the Web [17], as shown in figure 1. Because the information agents in this model are built as autonomous computational software entities, which have access to multiple, heterogeneous and geographically distributed information sources as in the Internet or corporate Intranet, they are called intelligent information agents (Section 3), and hence, the model can equally be described as the Intelligent Agent-Based Web Information Food Chain Model. These agents basically perform pro-active searches for, maintain, and mediate relevant information on behalf of their users or other agents preferably just-in-time.At the lowest level in the Web information food chain is raw content: the home pages of individuals and companies. The next level up the food chain is the services that 'consume' this raw content. These services include search engines such as Google, Lycos, and Yahoo. Search engines maintain large databases of web pages, indexed by content.

Apart from the technical difficulties associated with storing such large databases and being able to process and retrieve their contents sufficiently quickly to provide a useful online service, the search engines must also obtain and index new or changed web pages on a regular basis. Currently this is done in one or two ways. The simplest is to have human search for pages and classify them manually. This has the advantage that classification obtained in this way are likely to be meaningful and useful. But it has the very obvious disadvantage that it is not necessarily thorough, and is costly in terms of human resources. The second approach is to use simple software agents, often called spiders, to systematically search the web, following all links, and automatically classifying content. The classification of content is typically done by removing 'noise' words from page, and then attempting to find those words that have the most meaning.All current search engines, however, suffer from the disadvantage that their coverage is partial. A solution that was suggested as one way around this is to use a meta search engine. This search engine works not by directly maintaining a database of pages, but by querying a number of search engines in parallel. The result from this search engines can then be collated and presented to the user. The meta-search engine thus 'feeds' off the other search engines. By allowing running on the user's machine, it becomes possible to personalize services - to tailor them to the needs of individual users [17].
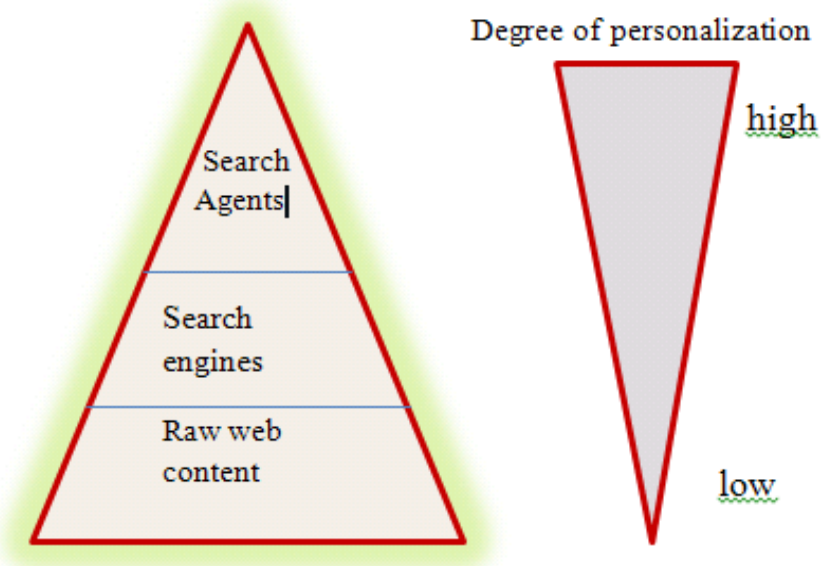

Figure 1: Intelligent Agent-Based Web Information Food Chain Model [17].

\section{B. Typical Architecture of a Multi-Agent Information System}

The information resources - Websites, are essentially passive. They simply deliver specific pages when requested. A common approach is thus to make information resources more 'intelligent' by wrapping them with agent capabilities. The structure of such a system is illustrated in figure 2 below.In this figure, theunderlying assumption isthat there are four (4) number of information repositories; Four (4) number of information agents; three (3) number of broker agents; and three (3) number of users. The information repositories may be websites, databases, or any other form of store. Access to these repositories is provided by information agents. These agents, which typicallycommunicate using an agent communication language, are 'experts' about their associated repository. As well as being able to provide access to the repository, they are able to answer 'meta-level' queries about the content ('do you know about X?'). The agent will communicate with the 
repository with whatever native API the repository provides HTTP, in the case of web repositories [17].

To address the issue of finding agents in an open environment like the internet, middle agents or brokers are used [17]. Each agent typically advertises its capabilities to some brokers. Brokers come in several different types. They may be simply matchmakers or yellow page agents, whichmatch advertisements to request for advertised capabilities. Alternatively, they may be blackboard agents, which simply collect and make available requests, or they may do both of these [17].

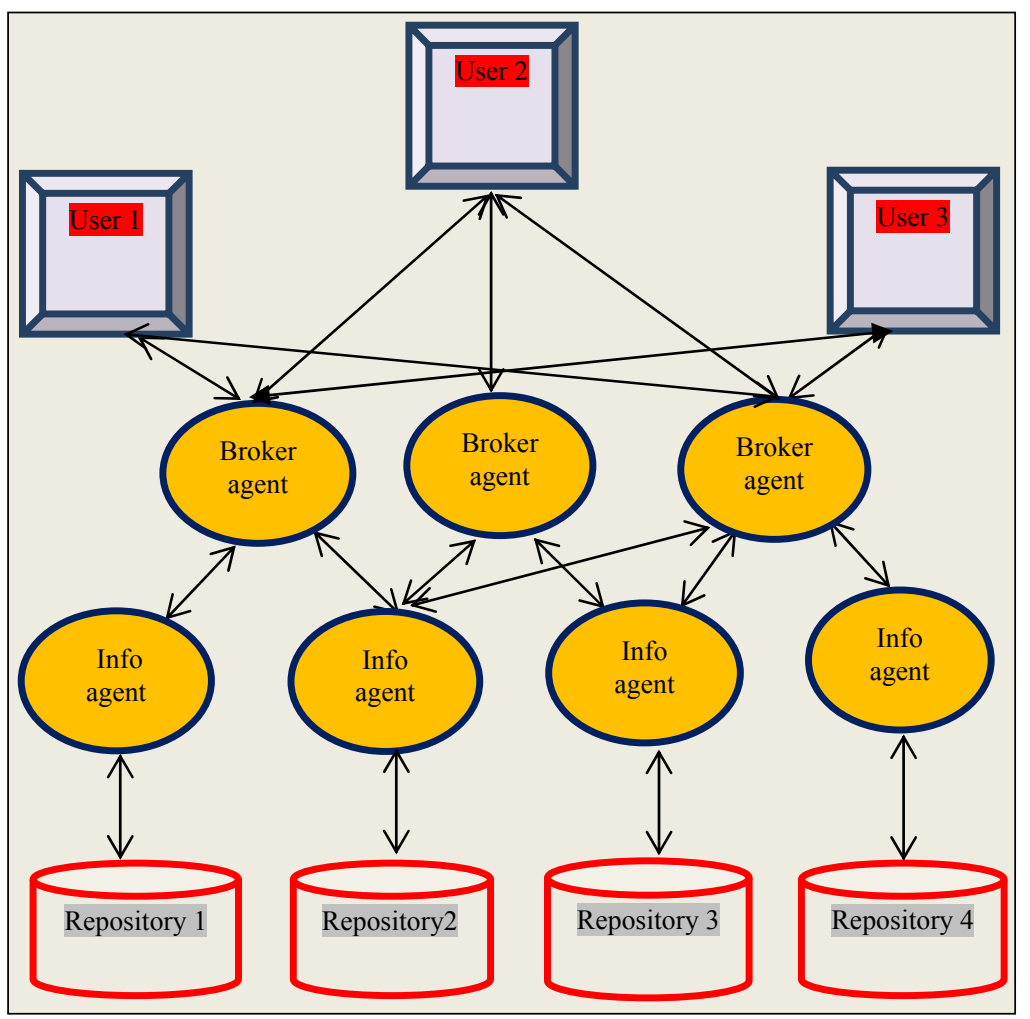

Figure2:Architecture of a Multi-Agent Information System [17]

Different brokers may be specialized in different areas of expertise, for example knowing about different repositories. Brokered systems are able to cope more quickly with a rapidly fluctuating agent population. Middle agents allow a system to operate robustly in the face of intermittent communications and agent appearance and disappearance. The overall behaviour of a system such as that in figure 3 is that, a user issues a query to an agent on their local machine. This agent may then contact information agents directly, or it may go to a broker, which is skilled at the appropriate type of request. The broker may then contact a number of information agents, asking first whether they have the correct skills, and then issuing specific queries. This kind of approach has been successfully used in digital library applications [10].

\section{Methodology for the Proposed Architecture for}

\section{Improved Collaborative Learning}

\section{Modification and Adoption of Web Information Model into IIFCEMAF}

In this proposed architecture, the underlying assumption is that there are $M$ number of information sources or repositories representing various application subjects or courses, $N$ number of learners, and brokers with a reduced number less than $N$, because each broker can serve as many users as possible within a specific geographical area. This is to reduce information overload' to the barest minimum. The architecture for the proposed collaborative IIFCEMAF has shown in Figure 3, with several mediating service brokers that have bidirectional communications between information agents and learners. Here, there is interaction within the service brokers regardless of which broker is directly connected to which service or user, with the brokers using distributed matching and multicasting techniques to handling and making communication possible and easy amongst millions of users and services. As shown in Figure 3, all users are connected through the interactions of the brokers and bidirectional communications between the subject application services (i.e. various sub-applications of IIFCEMAF) and learning Smartphone users. As a result, when a request or subscription is made by any user, the information is made available to the requesting user through another user via a designated or attached service broker connecting the Server and the users. Thus information is shared amongst the IIFCEMAF Smartphone holders. For example, if user $K(1 \leq K \leq N)$ makes a request or query to the Server, and the service repository holding such information could be another sub-application $M$ (say Maths. or Philosophy), the information can be made available through a concerned broker $P$ attached to $M$, which in turn notifies subscriber $K$, and makes the information obtained from $M$ available to $K$. The technology that supports this IIFCEMAF arrangement is shown in Figure 3 below.

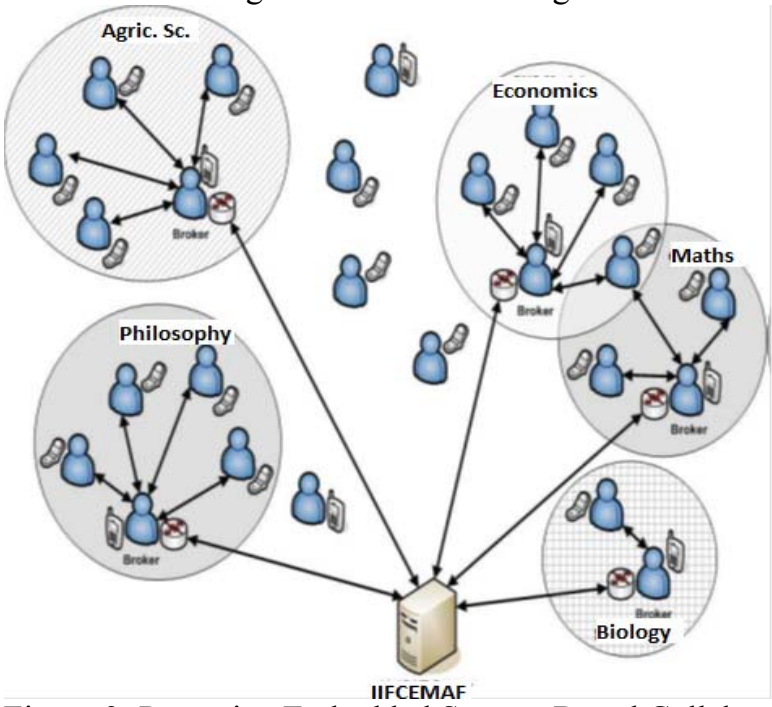

Figure 3: Pervasive Embedded System-Based Collaborative IIFCEMAF Architecture. 
Thus, there is intercommunication among the various subject repositories, learning users and brokers. This arrangement makes it possible for as many sub-application modules as possible that can be built into the IIFCEMAF system, accessed, and the information therein shared by collaborating users, whether subscribed or unsubscribed data plan.

\section{A. Application of Pervasive Embedded Systems into Collaborative IIFCEMAF Architecture.}

The Pervasive computing devices enable people to gain immediate access to information and services anywhere, anytime, without having to carry around heavy and impractical computing devices. Thus, the bulky PCs become less attractive and being slowly eroded with the development of a new generation of smart devices like wireless PDAs, smart phones, etc. These embedded devices are characterized by being unobtrusively embedded; completely connected; intuitively intelligent; effortlessly portable and mobile; and constantly on and available [8].

\section{B. Discussions}

Most of the existing collaborative systems without the embedded architecture remain with few weaknesses since majority of learning users are students, and sometimes do not subscribe to any data plan, even though smartphones are cheap and relatively common now. Many sometimes remain as offline users, and update their information only when they have $\mathrm{WiFi}$ connectivity. The mentioned weaknesses include:

- Data may become outdated.

- Not all users can share their status and progress instantly.

- Users without internet connectivity may miss out some important real-time information during or outside of lectures.

As compared to moste-learning systems, the proposed IIFCEMAF system is definitely provided with a more scalable and convenient solution. In our qualitative comparison, the proposed IIFCEMAF system provides "better" solution with the adoption of the Embedded System-based intelligent Web Information Chain Multi-Agent System framework into mobile in order to have an improved performance to collaborating users' anywhere, anytime. The enhancement eliminates the current limitation by allowing:

- The users without internet access or data plan subscription to utilize wireless connectivity (e.g. Bluetooth or ZigBee)

to detect the SNS users in the surrounding which have internet connectivity as proxy (service broker) for receiving and transmitting important information through his embedded system into the server.

- The learning users (students and lecturers) to share/broadcast progress information in a particular discourse or in as many subjects as possible with other collaborating users even though without direct internet access.

- An increase in the global e-learning capability amongst all users in order to share real-time information by utilizing wireless sensor network technology, which of courses increase the knowledge base of users all over the world.

However, the proposed IIFCEMAF has some possible weaknesses associated with it. For instance, users without data plan subscription may abuse the internet access which is provided by the service broker. They may overload the broker as a proxy for their internet connectivity. In order to solve this issue, we have designed the communication channel between users and brokers which only allowed small packet of important data (i,e, status, GPS location, short messages, sub-apps notification and reminder) to be shared across the e-learning users.

The aforementioned weaknesses notwithstanding, the adoption of Web Information model provides better solutions as a result of so many other valuable benefits it offers. First, the model has very simple interface, as shown in Figure 1. Other benefits are summarized below:

- Supports Large scale Mobile Phones: It provides a good platform for the use of very large number of devices, mobile phones and mobile applications available on these arrangements.

- Network Tolerance: Unreliable network connections associated with most current e-learning arrangements are tolerated with pervasive devices such that applications must continue to operate (in limited ways) while the network is unavailable and "recover" when the network is available.

- Sophisticated Interaction: The improved collaborative interaction pattern is brought about in pervasive mobile framework in unpredictable ways, that make mobile applications communicate with any number of mobile devices within proximity.

- Location support: This arrangement supports a communication abstraction that may make one application require addressing the services in a location-independent manner, while another may require location-dependent names.

- Data volume: The IIFCEMAF infrastructure supports the immense volume of data being transferred among the large number of mobile devices. Applications accessed by the individual devices use a simple and powerful mechanism to filter and process these data.

\section{Conclusion and Future Works}

The open Internet and worldwide Web allow us to access multimedia data and knowledge located throughout the world. Clearly, these new technologies present enormous opportunities for posting, finding, organizing and sharing vast amounts of information. Agent technology is the perfect way to model an autonomous process because of its ability to become an artificial society based on real-life society. This framework proposed in this article, has a Web Information Food Chain 
Model using an embedded multi layered agent architecture, targeted to improving collaborative e-learning across all spheres since education is a social service to be enjoyed by all, This framework, if adopted, applied and implemented without any form of conservatism or reservation, has a high potential of bringing about a better Multi-Agent Information Retrieval and Management System that enables users to find the precise information they really want to find, and shield them from information they do not want.With the infrastructural facilities in the Web Information Food Chain Multi-Agent Framework, we provide a better solution to the existing problem by making a unified access for improved collaborative learning by all users through Smartphone. This Mobile framework also enables users without data plan subscription, to receiving and transmitting important information, thus having access to learning updates alongside other users with data plan subscription. With this proposed arrangement, learning becomes so easily brought to the doorsteps of almost all citizens, especially between teachers and students, improves and encourages collaborative learning amongst all, especially in educational systems. However, the development of pervasive computing systems, whether embedded in the world around us or worn on the human body, is not without challenges. Future work should consider and take care of updating the intelligent agents dynamically using appropriate models to reflect the reality at any particular time since we are in a changing world. Also, acceptance can only be achieved if the technology is useful, convenient and unobtrusive, hence efforts should equally be improved upon to always remove almost perfectly the obstacles to the adoption of pervasive technology. Even though, the Belief-Desire-Intention (BDI) model has been proven to be a very efficient agent development model, efforts should also be made to 'combat' its observed limitations or shortcomings as they manifest or arise.

\section{References}

[1] BotSpot. A Collection of Bots in the Web. http://www.botspot.com/

[2] C.M. Bowman, "Harvest: A scalable, customizable discovery and access system", Technical Report, CU-CS 732-94, Uni Colorado, Boulder, USA. 1994.

[3] W.K. Cheng and H.Y.Chan, "Conflict Resolution in Resource Federation with Intelligent Agent",Negotiation". Practical Applications of Agent-based Technology. ISBN 978-953-51-02762,InTech.,2012.

[4] M. M.Chiu, "Group problem solving and individual actions". Journal for the Theory of Social Behavior, 30, 127 - 50. 600-631. 2000.

[5] K. Decker, K. Sycara, and M. Williamson, "Intelligent adaptive information agents". Journal on Intelligent Information Systems, Vol. 9, 239-260, 1997.

[6] P. Dillenbourg, "Collaborative Learning: Cognitive and Computational Approaches". Advances in Learning and Instruction Series. New York, NY: Elsevier Science, Inc.,1999.
[7] J.E. Doran, S. Franklin, N.R. Jennings, and T.J Norman, "On Cooperation in Multi-AgentSystems". The Knowledge Engineering Review,Vol. 12, 3rd Edition, 1997.

[8] G. Elliot and N. Phillips, "Pervasive and embedded mobile systems", Mobile Commerce and Wireless Computing Systems. Pearson Education Ltd. England, Pp. 363- 401, 2004.

[9] [E.O. Faleye, A.A. Ileladewa, and T.A. Ramoni, "Electronic learning”, Web Technology Made Easy. Aseda Publishing. Ibadan, Nigeria, pp. 274-284, December 2010.

[10] [Firefly network. http://www.firefly.com/

[11] K. Fischer, "Agent- based design on holonic manufacturing systems", Journal of Robotics and Autonomous Systems, 27: 313, 1999

[12] [V. Kashyap and A. Sheth, "Semantic information brokering". Proceedings Conference on Knowledge and Information Management CIKM, 1994.

[13] M. Klusch (Ed), "Intelligent Information Agents", Springer, 1999. M. Klusch, "Information Agent Technology for the Internet: A Survey". German Research Centre for Artificial Intelligence, Deduction and Multi-Agent Systems Lab, Germany. Pp. 1-33. 2001.

[14] R. Mitnik, Recabarren,, M. Nussbaum, and A. Soto, "Collaborative Robotic Instruction: A Graph Teaching Experience". Computers \& Education, Vol. 53, 2nd Edition, 330-342, 2009.

[15] G. WeiB (Ed). "Multiagent Systems”, MIT Press, 1999.

[16] M. Wooldridge, "Agents for information retrieval and management". An Introduction to Multi-Agent Systems. A John Wiley and Sons Publication, United Kingdom, 2nd Edition, pp 205210, 2009

Author:

A.A. Ileladewa
Department of Computer Science
Faculty of Information and Communication Technology
Universiti Tunku Abdul Rahman (UTAR)
Jalan Universiti, Bandar Barat
31900 Kampar, Perak
\{ileladewaadeoye@1utar.edu.my\}

